\title{
Eine Fields-Medaille für Elon Lindenstrauss
}

\section{Journal Article}

\section{Author(s):}

Einsiedler, Manfred

Publication date:

2011

Permanent link:

https://doi.org/10.3929/ethz-b-000422891

\section{Rights / license:}

In Copyright - Non-Commercial Use Permitted

\section{Originally published in:}

Mitteilungen der Deutschen Mathematiker-Vereinigung 19(1), https://doi.org/10.1515/dmvm-2011-0012 


\title{
Eine Fields-Medaille für Elon Lindenstrauss
}

\author{
Manfred Einsiedler
}

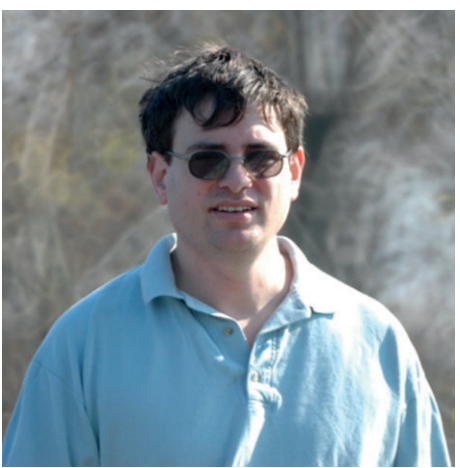

Elon Lindenstrauss

Elon Lindenstrauss hat vergangenen Sommer im Rahmen der Eröffnungszeremonie der ICM 2010 in Hyderabad Indien eine der vier Fields-Medaillen verliehen bekommen. Die Begründung des Preiskommittee für die Entscheidung war ,... for his results on measure rigidity in ergodic theory and their applications to number theory". Wir wollen hier kurz seine wichtigsten Arbeiten erläutern und auch Elon Lindenstrauss als Person vorstellen.

Elon ist in Jerusalem als Sohn von Joram Lindenstrauss, Mathematik-Professor an der Hebrew University, aufgewachsen. Man kann fast von einer modernen Mathematiker-Dynastie sprechen: Eine seiner Schwestern und ihr Mann sind Mathematiker an der Indiana University in Bloomington.

Elon hat an der Hebrew University in Jerusalem studiert und 1999 mit Benjamin Weiss als Betreuer promoviert. Elons erste Arbeiten beschäftigen sich mit dem Gebiet der Ergodentheorie.

In der Ergodentheorie geht es um statistische Fragen im Gebiet der Dynamischen Systeme und unter anderem um die mathematischen Grundlagen (und korrekte Formulierung) von Boltzmanns Ergodenhypothese. Die einfachste Form eines dynamischen Systems erhält man, indem man eine Abbildung $T: X \rightarrow X$ auf einem Raum $X$ betrachtet. Eine typische Frage besteht dann darin, die Bahn $x, T(x), T^{2}(x), \ldots$ eines Punktes $x \in X$ zu beschreiben. Hier wird $X$ der Zustandsraum (oder Phasenraum) genannt; z. B. könnte $X$ aus der Menge aller Positionen und Geschwindigkeiten von Sonne, Erde und Mond bestehen. In einer ersten Näherung, ohne Eigenrotationen einzubeziehen, ergibt sich in diesem Beispiel ein 18-dimensionaler Raum für $X$. Ein weiteres Beispiel für $X$ besteht aus Positionen und Geschwindigkeiten von allen Atomen in einem abgeschlossenen Raum. In beiden Beispielen ist die Abbildung durch die Zeitentwicklung gegeben: Für einen derzeitigen Zustand $x \in X$ ist $T(x)$ der Zustand nach einer (fest gewählten) Zeiteinheit. Das einfachste mathematische Modell eines dynamischen Systems besteht aus dem halb-offenen Intervall $X=[0,1)$ und der Abbildung $R_{\alpha}(x)=\lfloor x+\alpha\rfloor$, die den Punkt $x$ auf den Nachkommaanteil der Summe $x+\alpha$ für ein festes $\alpha \in \mathbb{R}$ abbildet.

Einer der grundlegendsten Sätze der Ergodentheorie ist der Ergodensatz von Birkhoff: In einem ergodischen System, welches aus einer Abbildung $T: X \rightarrow X$ auf einem Raum $X$ und einem invarianten $\mathrm{Ma} \beta \mu$ auf $X$ (in anderen Worten einer stationären Integrationsmethode von Funktionen auf $X$ ) besteht, gilt, dass der Grenzwert

$$
\lim _{N \rightarrow \infty} \frac{1}{N} \sum_{n=1}^{N} f\left(T^{n}(x)\right)
$$

für jede integrierbare Funktion $f$ und $\mu$-fast jedem $x \in X$ existiert. Hier sollte das ,fast jedes $x$ “ so interpretiert werden: Es kann zwar sein, dass es Punkte gibt, an denen obiger Grenzwert nicht existiert, aber statistisch gesehen sind solche Punkte vernachlässigbar. Boltzmanns Ergodenhypothese besagte ursprünglich, dass die Bahn eines Punktes $x, T(x), T^{2}(x), \ldots$ jeden anderen Punkt mit derselben Energie erreicht. Dies ist aus mengentheoretischen Gründen (und wenn man kontinuierliche Zeit zulässt aus topologischen Gründen) nicht möglich, die mathematisch korrekte Formulierung dieses grundlegenden Konzeptes ist folgendermaßen: Falls es keine nichttrivialen invarianten Teilmengen gibt (d. h. alle messbaren invarianten Teilmengen von $X$ haben bezüglich $\mu$ Maß 0 oder I), dann sagt man, $\mu$ is ergodisch. Falls $\mu$ ergodisch ist, dann ist der Grenzwert von (I) durch $\int f d \mu$ gegeben, und man erhält in diesem Sinn, dass das Mittel über die Zeit gleich dem Mittel über den Raum ist. Zum Beispiel ist die Abbildung $R_{\alpha}$ genau dann ergodisch bezüglich dem Lebesgue-Maß falls $\alpha$ eine irrationale Zahl ist.

Obwohl dieser Satz sehr allgemein ist und sehr viele verschiedene Fälle für den Raum $X$, die Abbildung $T$ oder das $\mathrm{Maß} \mu$ zulässt, gibt es doch Anwendungen (sowohl in der Physik und der Zahlentheorie), die Verallgemeinerungen benötigen. Eine verallgemeinerte Theorie erhält man, wenn man anstatt einer einzigen Transformation $T$ eine ganze Gruppe $G$ von Transformationen auf $X$ zulässt. Zum Beispiel könnte $G \cong \mathbb{R}^{d}$ sein, und statt ein Zeitmittel von 1 bis $N$ wie in (I) zu betrachten, könnte man über einen $d$-dimensionalen Würfel $[0, L]^{d}$ der Länge $L$ mitteln. Der Ergodensatz von Birkhoff gilt auch für $G \cong \mathbb{R}^{d}$ und ist in diesem und auch anderen Spezialfällen seit Langem bekannt. Nicht in jeder Gruppe kann man solche Zeitmittel betrachten, aber eine sehr natürliche Klasse von Gruppen, auf denen man das Zeitmittel mit ähnlichen Eigenschaften definieren kann, ist die Klasse 
der mittelbaren Gruppen. Elon hat in einer seiner ersten Arbeiten [8] den allgemeinen Ergodensatz für jede mittelbare Gruppe bewiesen.

Kurz nach seinem Studium ging Elon in die USA, wo er zuerst als Postdoc am Institute for Advanced Studies (IAS) in Princeton, dann in Stanford, am Courant Institute an der NYU in New York, und schlussendlich von 2004 bis 2009 an der Princeton University als Professor tätig war. Am Institute for Advanced Studies begann Elon sich mit Quantum Chaos und Starrheitssätzen für invariante Maße zu beschäftigen. Im gleichen Jahr ging ich auch in die USA, genauer gesagt an die Penn State, und auch ich arbeitete gemeinsam mit Anatole Katok an derartigen Starrheitssätzen. Elon und ich haben uns dann an der ETH in Zürich während einer Konferenz erstmals getroffen, und er hat mich eingeladen, ihn in Stanford zu besuchen. Dort haben wir unsere langjährige Zusammenarbeit begonnen.

Elon ist in der Mathematik sehr zielstrebig. In unserer Zusammenarbeit hält er oft hartnäckig an seinem Glauben fest, dass sich etwas Neues zu dem gegebenen Problem beweisen lässt, selbst wenn wir uns schon wochenlang (oder jahrelang) die Köpfe zerbrochen haben. Sein Beweis für die Starrheit der invarianten Maße für den geodäsischen Fluss (,low entropy“ method) in [9] aus seiner Zeit am IAS kann nur aus dieser Hartnäckigkeit resultieren. Ich kann mich gut errinnern, wie ich zum ersten Mal diesen Beweis durchgelesen habe und es lange nicht glauben konnte, dass sich am Ende der Knoten auflösen wird. Aber zu guter Letzt war ich überzeugt. Kurz nach dieser Arbeit haben wir die Methode gemeinsam mit Anatole Katok von $\mathrm{SL}_{2}(\mathbb{R})$ auf $\mathrm{SL}_{n}(\mathbb{R})$ verallgemeinert, siehe [4]. Die endgültige Version der Methode (die technisch nochmals anspruchsvoller war) erschien dann 2008 in einer gemeinsamen Arbeit [5] von Elon und mir. In diesen Arbeiten geht es darum, invariante Maße für den geodäsischen Fluss oder allgemeineren Gruppen von Transformationen auf homogenen Räumen zu beschreiben. Idealerweise will man beweisen dass das untersuchte invariante Maß das Volumenmaß ist. Siehe [3] für weitere einführende Erläuterungen zu dem Thema Starrheit der invarianten Maße.

Ein weiteres Kennzeichen von Elons Arbeiten sind die zahlreichen Verknüpfungen von Ideen aus verschiedenen Teilgebieten der Mathematik. In unserer gemeinsamen Arbeit [4] mit Anatole Katok haben wir zum Beispiel die Starrheit der invarianten Maße verwendet, um folgendes Resultat in dem Gebiet der Diophantischen Approximation zu beweisen. Littlewood hat um 1930 vermutet, dass man für je zwei reelle Zahlen $\alpha_{1}, \alpha_{2} \in \mathbb{R}$ rationale Approximationen $\frac{p_{1}}{q}, \frac{p_{2}}{q}$ finden kann, so dass das Produkt

$$
q^{3}\left|\alpha_{1}-\frac{p_{1}}{q}\right|\left|\alpha_{2}-\frac{p_{2}}{q}\right|
$$

beliebig klein gemacht werden kann. Es ist relativ leicht zu zeigen, dass (Lebesgue-)fast alle Paare $\left(\alpha_{1}, \alpha_{2}\right)$ dies erfüllen. Mit der Starrheit der invarianten Maße konnten wir zeigen, dass die Menge der Paare, die die Vermutung nicht erfüllt, höchstens Hausdorff-Dimension null haben muss. Die leere Menge ist hier nicht ausgeschlossen, und die Vermutung ist trotz dieses Teilerfolges noch offen.

Das für mich beste Beispiel einer solchen Verknüpfung von Ideen aus verschiedenen Gebieten der Mathematik sind die Arbeiten von Elon und Jean Bourgain an der Quantum Unique Ergodicity Vermutung von Rudnick und Sarnak. Es geht hier um die elementaren Schwingungen auf hyperbolischen Flächen (die Verallmeinerungen der Sinus-Schwingungen auf einem Intervall), genauer gesagt die Eigenfunktionen des hyperbolischen LaplaceOperators. In Sinne der Quantum Physik interpretiert man $|\phi|^{2}$ für eine Eigenfunktion $\phi$ als die Dichteverteilung eines Quantum-Teilchens. Die Vermutung besagt, dass die Verteilung gegen die Gleichverteilung auf der Fläche strebt, wenn der Eigenwert (die Energie des Teilchens) gegen unendlich strebt. Elon beweist diese Aussage in seiner Arbeit [9] unter der zusätzlichen Annahme, dass die Eigenfunktionen auch Eigenfunktionen der zahlentheoretischen Hecke-Operatoren sind. Er erreicht dies, indem er die oben erwähnte (,low entropy“) Form der Starrheit der invarianten Maße zeigt und dann die notwendigen Vorraussetzungen für diesen Satz beweist, eine der Vorrausetzungen ist in der gemeinsam Arbeit [2] mit Jean Bourgain gezeigt. Hier spielt jedes der Gebiete Darstellungstheorie, Zahlentheorie und eben Ergodentheorie eine entscheidende Rolle, siehe auch [7].

Ich will noch zwei Beispiele für solche Verknüpfung von Ideen aufführen. In der gemeinsamen Arbeit [I] von Jean Bourgain, Alex Furman, Elon und Shahar Mozes verwenden die Autoren Sätze aus Additiver Kombinatorik (sumproduct-phenomenon), um einen Satz in Ergodentheorie zu beweisen. In der gemeinsamen Arbeit [6] von Elon, Philippe Michel, Akshay Venkatesh und mir verwenden wir wiederum den Starrheitssatz aus [4] und bekannte Subkonvexitätssätze der Dedekind $\zeta$-Funktionen eines Zahlenkörpers, um die Gleichverteilung von Idealklassen von kubischen Zahlenköpern zu beweisen.

In 2009 trat Elon eine Professur an der Hebrew University in Jerusalem an. Er lebt jetzt dort gemeinsam mit seiner Frau und drei Töchtern in seinem Haus in der Nähe der Universität und nur wenige Gehminuten von seinem Elternhaus entfernt.

\section{Literatur}

[I] J. Bourgain, A. Furman, E. Lindenstrauss, S. Mozes. Stationary measures and equidistribution for orbits of nonabelian semigroups on the torus. J. Amer. Math. Soc. 24 (20I I), no. I, 23I-280.

[2] J. Bourgain, E. Lindenstrauss. Entropy of quantum limits. Comm. Math. Phys. 233 (2003), no. I, I53-171.

[3] M. Einsiedler. What is ... measure rigidity? Notices Amer. Math. Soc. 56 (2009), no. 5, 600-60I. 
[4] M. Einsiedler, A. Katok, E. Lindenstrauss. Invariant measures and the set of exceptions to Littlewood's conjecture. Ann. of Math. (2) 164 (2006), no. 2, 5I3-560.

[5] M. Einsiedler, E. Lindenstrauss. On measures invariant under diagonalizable actions: the rank-one case and the general low-entropy method. J. Mod. Dyn. 2 (2008), no. I, 83-128.

[6] M. Einsiedler, E. Lindenstrauss, Ph. Michel, and A. Venkatesh. Distribution of periodic torus orbits and Duke's theorem for cubic fields. To appear, Annals of Math.

[7] M. Einsiedler, T. Ward. Arithmetic Quantum Unique Ergodicity for $\Gamma \backslash \mathbb{H}$. http://math.arizona.edu/ swc/aws/I0/ 2010 EinsiedlerNotes.pdf

[8] E. Lindenstrauss. Pointwise theorems for amenable groups. Electron. Res. Announc. Amer. Math. Soc. 5 (1999), 82-90 (electronic).

[9] E. Lindenstrauss. Invariant measures and arithmetic quantum unique ergodicity. Ann. of Math. (2) 163 (2006), no. I, I65-2I9.
Prof. Dr. Manfred Einsiedler, ETH Zürich,

Departement Mathematik, Rämistraße I0I, 8092 Zürich

manfred.einsiedler@math.ethz.ch

Manfred Einsiedler hat 1999 in Mathematik an der Universität Wien promoviert. Schwerpunkt seines Studiums war die Theorie der mehrparametrischen Dynamik. Von 200I bis 2009 arbeitete er an mehreren Universitäten (Penn State University, Washington State University, Princeton University, Ohio State University) in den USA. 2009 kehrte Manfred Einsiedler nach Europa zurück, um eine Professor an

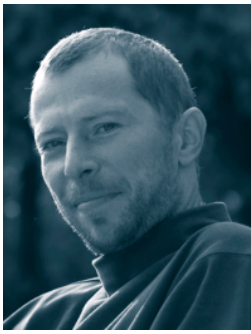
der ETH Zürich anzutreten.

\section{Eine Fields-Medaille für Cedric Villani}

Felix Otto

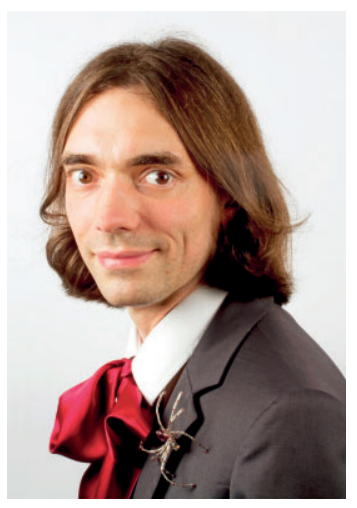

Cedric Villani (Foto: Pierre Maraval)

Angefangen mit der Dissertation über die Habilitation bis hin zu der Arbeit, für die Cedric Villani mit der Fields-Medaille ausgezeichnet wurde, zieht sich ein Thema wie ein roter Faden durch die meisten seiner Veröffentlichungen: Was ist der Ursprung und das Wesen von Reibung in Flüssigkeiten, Gasen und Plasmen? Diese Frage ist gleichsam von philosophischem Interesse: Obwohl die einzelnen Partikel, z. B. Gasmoleküle oder Elektronen, der zeitumkehrbaren Newton'schen Mechanik (oder gar der Quantenmechanik) genügen, verhält sich das gesamte Medium effektiv so, als wäre die Zeit nicht umkehrbar. Zum Beispiel streben räumlich lokal gemittelte Größen, etwa die Geschwindigkeitsverteilung, einem Gleichgewicht entgegen. Aus mathematischer Sicht stellt sich die Frage wie folgt: Wie entsteht aus einem zeitumkehrbaren (riesigen) System von gewöhnlichen Differentialgleichungen ein effektiv irreversibles Verhalten für gemittelte Größen? Die mathematische Phantasie und hartnäckige Konsequenz, mit der sich Villani dieser Fragestel- lung widmet, hat mich schon beeindruckt, als ich ihn kurz nach seiner Promotion kennenlernte.

\section{Die Boltzmanngleichung und der Trend zum Gleichgewicht}

Ein fundamentales Modell, anhand dessen Villani diese Frage studiert hat, ist durch die Boltzmanngleichung gegeben. Die Boltzmanngleichung liegt auf halbem Wege zwischen den Newton'schen Bewegungsgleichungen für die Fluidpartikel und den Navier-Stokes-Gleichungen für viskose Fluide. In Letzteren ist die Reibung (d.h. innere Reibung oder Viskosität) und damit die Irreversibilität und der Trend zum Gleichgewicht explizit eingebaut.

Zunächst scheint es so, als wäre die Boltzmanngleichung ein getreues Abbild der Newton'schen Mechanik - nur auf einer anderen Beschreibungsebene: Statt den Zustand durch die Positionen $\left\{X_{i}\right\}_{i=1, \cdots, N}$ und die Geschwindigkeiten $\left\{V_{i}\right\}_{i=1, \cdots, N}$ der (idealisiert kugelförmigen und identischen) $N \gg 1$ Teilchen zu beschreiben, wird der Zustand durch eine Anzahldichte $F(t, x, v) d x d v$ im Phasenraum von Orts- und Geschwindigkeitskoordinaten $\mathbb{R}^{3} \times \mathbb{R}^{3}$ beschrieben: Durch Integration der Dichte $\int_{A} F(t, x, v) d x d v$ erhält man also die Gesamtzahl der Teilchen zu einem Zeitpunkt $t$ mit Position und Geschwindigkeit in einer Teilmenge $A$ des Phasenraums. Die Boltzmanngleichung hat die einfache Form:

$$
\begin{aligned}
\partial_{t} F(t, x, v)+v \cdot \nabla_{x} F(t, x, v) \\
=Q(F(t, x, \cdot), F(t, x, \cdot))(v) .
\end{aligned}
$$

